Supplementary data for manuscript

\title{
SYNTHESIS OF FUNCTIONALIZED N-SULFONYLAMIDINES FROM N-SULFONYLKETENIMINES AND 2-AMINOBENZIMIDAZOLE
}

\author{
Issa Yavari, Azam Sheikhi, Manijeh Nematpour, and Zohreh Taheri \\ Department of Chemistry, Tarbiat Modares University, Tehran, Iran
}

GRAPHICAL ABSTRACT

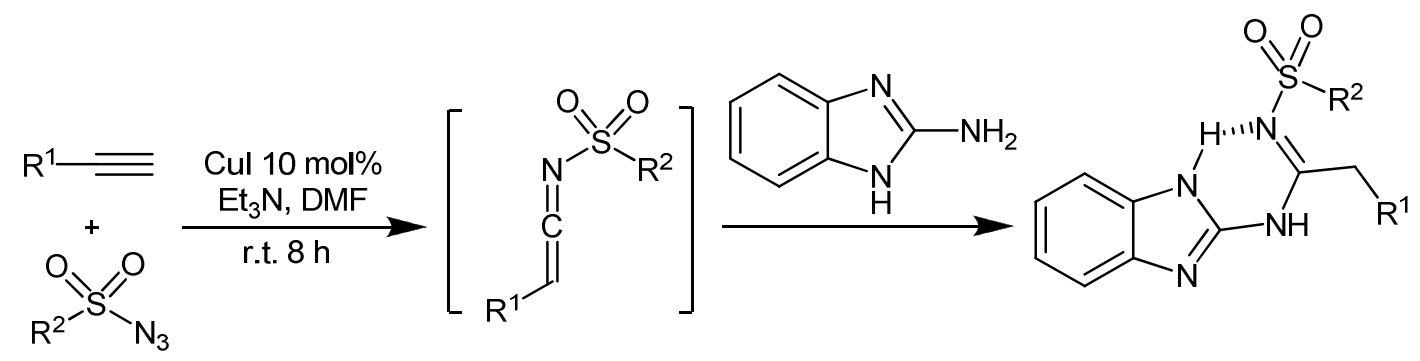

\begin{abstract}
The synthesis of a novel class of $N$-(1H-benzo[d]imidazol-2-yl)-2-alkyl-N'sulfonylacetamidines via a copper-catalyzed three-component coupling reaction of $1 H$ benzo[d]imidazol-2-amine, sulfonyl azides and terminal alkynes is described.
\end{abstract}

Keywords Amidines; copper iodide; $N$-sulfonylketenimines; tosyl azide; terminal alkyne

Address correspondence to Issa Yavari, Department of Chemistry, Tarbiat Modares University, PO Box 14115-175, Tehran, Iran. E-mail: yavarisa@modares.ac.ir 


\section{EXPERIMENTAL}

All chemicals were obtained commercially and used without further purification. M.p.: Electrothermal-9100 apparatus. IR Spectra: Shimadzu-IR-460 spectrometer; bond positions in $\mathrm{cm}^{-1} .{ }^{1} \mathrm{H}-$ and ${ }^{13} \mathrm{C}-\mathrm{NMR}$ Spectra: Bruker DRX-500 Avance instrument at 400.1 and $100.6 \mathrm{MHz}$, resp.; $\delta$ in ppm, $J$ in Hz. MS: Finnigan-MAT-8430EI-MS mass spectrometer; at $70 \mathrm{eV}$; in $\mathrm{m} / \mathrm{z}$ (rel. \%). Elemental analyses: Vario EL III CHNOS elemental analyzer.

\section{General Procedure for the Preparation of Compounds 5}

To a mixture of alkyne 1 (1 mmol), sulfonyl azide 2 (1.2 mmol), CuI (0.1 mmol), and $\mathrm{Et}_{3} \mathrm{~N}(1 \mathrm{mmol})$ in DMF $(3 \mathrm{~mL})$ was slowly added 2-amino-benzoimidazole $(1.5 \mathrm{mmol})$. The mixture was stirred at room temperature. After completion of the reaction [about $8 \mathrm{~h}$; TLC (AcOEt/hexane 1:3) monitoring], the mixture was diluted with $\mathrm{CH}_{2} \mathrm{Cl}_{2}(2 \mathrm{~mL})$ and aqueous $\mathrm{NH}_{4} \mathrm{Cl}$ solution ( $3 \mathrm{~mL}$ ), stirred for $30 \mathrm{~min}$, and the layers were separated. The aqueous layer was extracted with $\mathrm{CH}_{2} \mathrm{Cl}_{2}(3 \mathrm{~mL} \times 3)$ and the combined organic fractions were dried $\left(\mathrm{Na}_{2} \mathrm{SO}_{4}\right)$ and concentrated under reduced pressure. The residue was purified by flash column chromatography [silica gel (230-400 mesh; Merck), AcOEt/hexane 1:3] to give product.

$\mathbf{N}$-(1H-benzo[d]imidazol-2-yl)-2-phenyl-N'-tosylacetamidine 5. Yield: $0.32 \mathrm{~g}(80 \%)$; cream powder; m.p. $=156-159{ }^{\circ} \mathrm{C} . \mathrm{IR}(\mathrm{KBr}): 3160(\mathrm{NH}), 3030(\mathrm{NH}), 1448(\mathrm{C}=\mathrm{N}), 1375\left(\mathrm{SO}_{2}\right)$, $1178 \mathrm{~cm}^{-1}\left(\mathrm{SO}_{2}\right) .{ }^{1} \mathrm{H}$ NMR $\left(400 \mathrm{MHz}, \mathrm{CDCl}_{3}\right): \delta_{\mathrm{H}}=2.48(3 \mathrm{H}, \mathrm{s}, \mathrm{Me}), 4.25\left(2 \mathrm{H}, \mathrm{s}, \mathrm{CH}_{2}\right), 7.29-7.35$ $(3 \mathrm{H}, \mathrm{m}, \mathrm{Ph}), 7.40\left(2 \mathrm{H}, \mathrm{d},{ }^{3} J_{\mathrm{HH}}=7.9 \mathrm{~Hz}, \mathrm{Ar}\right), 7.48\left(2 \mathrm{H}, \mathrm{d},{ }^{3} J_{\mathrm{HH}}=7.5 \mathrm{~Hz}, \mathrm{Ar}\right), 7.57\left(1 \mathrm{H}, \mathrm{t},{ }^{3} J_{\mathrm{HH}}=\right.$ $7.4 \mathrm{~Hz}, \mathrm{Ar}), 7.62\left(1 \mathrm{H}, \mathrm{t},{ }^{3} J_{\mathrm{HH}}=7.4 \mathrm{~Hz}, \mathrm{Ar}\right), 7.91\left(2 \mathrm{H}, \mathrm{d},{ }^{3} J_{\mathrm{HH}}=7.9 \mathrm{~Hz}, \mathrm{Ar}\right), 8.02\left(1 \mathrm{H}, \mathrm{d},{ }^{3} J_{\mathrm{HH}}=\right.$ $7.4 \mathrm{~Hz}, \mathrm{Ar}), 8.26\left(1 \mathrm{H}, \mathrm{d},{ }^{3} \mathrm{~J}_{\mathrm{HH}}=7.4 \mathrm{~Hz}, \mathrm{Ar}\right), 9.59(1 \mathrm{H}, \mathrm{s}, \mathrm{NH}), 10.48(1 \mathrm{H}, \mathrm{s}, \mathrm{NH}) .{ }^{13} \mathrm{C} \mathrm{NMR}$ $\left(100.6 \mathrm{MHz}, \mathrm{CDCl}_{3}\right): \delta_{\mathrm{C}}=30.8(\mathrm{Me}), 47.6\left(\mathrm{CH}_{2}\right), 123.3(\mathrm{CH}), 123.6(\mathrm{CH}), 127.2(\mathrm{C}), 127.9(\mathrm{CH})$, $128.2(2 \mathrm{CH}), 128.6(\mathrm{CH}), 129.5(2 \mathrm{CH}), 130.2(\mathrm{CH}), 130.8(\mathrm{C}), 131.4(2 \mathrm{CH}), 133.8(2 \mathrm{CH}), 141.7$ 
(C), $142.8(\mathrm{C}), 148.2(\mathrm{C}), 158.1(\mathrm{C}), 166.0(\mathrm{C})$. MS (EI, $70 \mathrm{eV}): \mathrm{m} / z(\%)=404\left(\mathrm{M}^{+}, 5\right), 328(10)$, 313 (13), 287 (16), 249 (32), 155 (100), 91 (70), 77 (54). Anal. calcd. for $\mathrm{C}_{22} \mathrm{H}_{20} \mathrm{~N}_{4} \mathrm{O}_{2} \mathrm{~S}$ (404.13): C, 65.33; H, 4.98; N, 13.85\%. Found: C, 65.69; H, 5.02; N, 13.94\%.

\section{$N$-(1H-benzo[d]imidazol-2-yl)-2-phenyl- $N^{\prime}$-(phenylsulfonyl)acetimidamide $5 \mathrm{~b}$.}

Yield: 0.32 g (82\%); cream powder; m.p. $=132-134{ }^{\circ} \mathrm{C} . \mathrm{IR}(\mathrm{KBr}): 3137(\mathrm{NH}), 3003(\mathrm{NH}), 1448$ $(\mathrm{C}=\mathrm{N}), 1376\left(\mathrm{SO}_{2}\right), 1181 \mathrm{~cm}^{-1}\left(\mathrm{SO}_{2}\right) .{ }^{1} \mathrm{H}$ NMR $\left(400 \mathrm{MHz}, \mathrm{CDCl}_{3}\right): \delta_{\mathrm{H}}=4.28\left(2 \mathrm{H}, \mathrm{s}, \mathrm{CH}_{2}\right), 7.30-$ $7.37(3 \mathrm{H}, \mathrm{m}, \mathrm{Ph}), 7.49\left(2 \mathrm{H}, \mathrm{d},{ }^{3} J_{\mathrm{HH}}=7.8 \mathrm{~Hz}, \mathrm{Ar}\right), 7.59\left(1 \mathrm{H}, \mathrm{t},{ }^{3} J_{\mathrm{HH}}=7.5 \mathrm{~Hz}, \mathrm{Ar}\right), 7.66-7.74(3 \mathrm{H}, \mathrm{m}$,

$\mathrm{Ph}), 7.77\left(1 \mathrm{H}, \mathrm{t},{ }^{3} J_{\mathrm{HH}}=7.5 \mathrm{~Hz}, \mathrm{Ar}\right), 8.00-8.06(3 \mathrm{H}, \mathrm{m}, \mathrm{Ph}), 8.29\left(1 \mathrm{H}, \mathrm{d},{ }^{3} \mathrm{~J}_{\mathrm{HH}}=7.5 \mathrm{~Hz}, \mathrm{Ar}\right), 9.72$ $(1 \mathrm{H}, \mathrm{s}, \mathrm{NH}), 10.88(1 \mathrm{H}, \mathrm{s}, \mathrm{NH}) .{ }^{13} \mathrm{C} \mathrm{NMR}\left(100.6 \mathrm{MHz}, \mathrm{CDCl}_{3}\right): \delta_{\mathrm{C}}=46.9\left(\mathrm{CH}_{2}\right), 122.9(\mathrm{CH}), 123.7$ $(\mathrm{CH}), 127.3(\mathrm{CH}), 128.0(2 \mathrm{CH}), 128.2(\mathrm{CH}), 128.7(\mathrm{CH}), 129.2(2 \mathrm{CH}), 130.3(\mathrm{CH}), 130.7(2 \mathrm{CH})$, 133.6 (2 CH), 134.5 (C), 136.4 (C), 145.6 (C), 148.7 (C), 158.7 (C), 167.1 (C). MS (EI, 70 eV): $\mathrm{m} / \mathrm{z}$ $(\%)=390\left(\mathrm{M}^{+}, 1\right), 313$ (6), 299 (10), 273 (18), 249 (16), 141 (100), 132 (21), 91 (38), 77 (50), 78 (31). Anal. calcd. for $\mathrm{C}_{21} \mathrm{H}_{18} \mathrm{~N}_{4} \mathrm{O}_{2} \mathrm{~S}$ (390.12): C, 64.60; H, 4.65; N, 14.35\%. Found: C, 64.93; H, $4.69 ; \mathrm{N}, 14.42 \%$.

\section{$N$-(1H-benzo[d]imidazol-2-yl)-N'-(methylsulfonyl)-2-phenylacetimidamide $5 \mathrm{c}$.}

Yield $0.24 \mathrm{~g}(74 \%)$; cream powder, m.p. $=122-124^{\circ} \mathrm{C} . \mathrm{IR}(\mathrm{KBr}): 3324(\mathrm{NH}), 3010(\mathrm{NH}), 1448$ $(\mathrm{C}=\mathrm{N}), 1369\left(\mathrm{SO}_{2}\right), 1176 \mathrm{~cm}^{-1}\left(\mathrm{SO}_{2}\right) .{ }^{1} \mathrm{H} \mathrm{NMR}\left(400 \mathrm{MHz}, \mathrm{CDCl}_{3}\right): \delta_{\mathrm{H}}=3.68(3 \mathrm{H}, \mathrm{s}, \mathrm{Me}), 4.35$ $\left(2 \mathrm{H}, \mathrm{s}, \mathrm{CH}_{2}\right), 7.31-7.35(3 \mathrm{H}, \mathrm{m}, \mathrm{Ph}), 7.50\left(2 \mathrm{H}, \mathrm{d},{ }^{3} \mathrm{~J}_{\mathrm{HH}}=7.8 \mathrm{~Hz}, \mathrm{Ar}\right), 7.60\left(1 \mathrm{H}, \mathrm{t},{ }^{3} \mathrm{~J}_{\mathrm{HH}}=7.5 \mathrm{~Hz}\right.$, Ar), $7.66\left(1 \mathrm{H}, \mathrm{t},{ }^{3} J_{\mathrm{HH}}=7.7 \mathrm{~Hz}, \mathrm{Ar}\right), 8.05\left(1 \mathrm{H}, \mathrm{d},{ }^{3} J_{\mathrm{HH}}=7.5 \mathrm{~Hz}, \mathrm{Ar}\right), 8.32\left(1 \mathrm{H}, \mathrm{d},{ }^{3} J_{\mathrm{HH}}=7.5 \mathrm{~Hz}\right.$, $\operatorname{Ar}), 9.77(1 \mathrm{H}, \mathrm{s}, \mathrm{NH}), 10.89(1 \mathrm{H}, \mathrm{s}, \mathrm{NH}) .{ }^{13} \mathrm{C} \mathrm{NMR}\left(100.6 \mathrm{MHz}, \mathrm{CDCl}_{3}\right): \delta_{\mathrm{C}}=30.8(\mathrm{Me}), 46.0$ $\left(\mathrm{CH}_{2}\right), 122.6(\mathrm{CH}), 123.2(\mathrm{C}), 123.9(\mathrm{CH}), 128.4(\mathrm{CH}), 129.2(\mathrm{CH}), 129.4(2 \mathrm{CH}), 130.0(\mathrm{CH})$, $133.2(2 \mathrm{CH}), 133.4(\mathrm{C}), 147.9(\mathrm{C}), 159.2(\mathrm{C}), 165.0(\mathrm{C})$. MS (EI, $70 \mathrm{eV}): m / z(\%)=328\left(\mathrm{M}^{+}, 5\right)$, 
252 (6), 273 (10), 211 (18), 196 (16), 91 (23), 78 (100), 77 (50). Anal. calcd. for $\mathrm{C}_{16} \mathrm{H}_{16} \mathrm{~N}_{4} \mathrm{O}_{2} \mathrm{~S}$ (328.10): C, 58.52; H, 4.91; N, 17.06\%. Found: C, 58.21; H, 4.95; N, 17.17\%.

$\mathbf{N}$-(1H-benzo[d]imidazol-2-yl)-N'-tosylhexanamidine 5d. Yield 0.24 g, (62\%); cream powder, mp: $111-113{ }^{\circ} \mathrm{C}, \mathrm{IR}(\mathrm{KBr}): 3224(\mathrm{NH}), 3039(\mathrm{NH}), 1490(\mathrm{C}=\mathrm{N}), 1368\left(\mathrm{SO}_{2}\right)$, $1179 \mathrm{~cm}^{-1}\left(\mathrm{SO}_{2}\right) .{ }^{1} \mathrm{H}$ NMR $\left(400 \mathrm{MHz}, \mathrm{CDCl}_{3}\right): \delta_{\mathrm{H}}=0.92\left(3 \mathrm{H}, \mathrm{t},{ }^{3} J_{\mathrm{HH}}=6.9 \mathrm{~Hz}, \mathrm{Me}\right), 1.39-1.45$ $\left(2 \mathrm{H}, \mathrm{m}, \mathrm{CH}_{2}\right), 1.48-1.54\left(2 \mathrm{H}, \mathrm{m}, \mathrm{CH}_{2}\right), 2.17-2.21\left(2 \mathrm{H}, \mathrm{m}, \mathrm{CH}_{2}\right), 2.49(3 \mathrm{H}, \mathrm{s}, \mathrm{Me}), 3.24(2 \mathrm{H}, \mathrm{t}$, $\left.{ }^{3} J_{\mathrm{HH}}=6.9 \mathrm{~Hz}, \mathrm{CH}_{2}\right), 7.41\left(2 \mathrm{H}, \mathrm{d},{ }^{3} J_{\mathrm{HH}}=7.9 \mathrm{~Hz}, \mathrm{Ar}\right), 7.64\left(1 \mathrm{H}, \mathrm{t},{ }^{3} J_{\mathrm{HH}}=7.5 \mathrm{~Hz}, \mathrm{Ar}\right), 7.72(1 \mathrm{H}, \mathrm{t}$, $\left.{ }^{3} J_{\mathrm{HH}}=7.5 \mathrm{~Hz}, \mathrm{Ar}\right), 7.91\left(2 \mathrm{H}, \mathrm{d},{ }^{3} J_{\mathrm{HH}}=7.9 \mathrm{~Hz}, \mathrm{Ar}\right), 8.08\left(1 \mathrm{H}, \mathrm{d},{ }^{3} J_{\mathrm{HH}}=7.5 \mathrm{~Hz}, \mathrm{Ar}\right), 8.37(1 \mathrm{H}, \mathrm{d}$, $\left.{ }^{3} J_{\mathrm{HH}}=7.5 \mathrm{~Hz}, \mathrm{Ar}\right), 9.39(1 \mathrm{H}, \mathrm{s}, \mathrm{NH}), 10.98(1 \mathrm{H}, \mathrm{s}, \mathrm{NH}) .{ }^{13} \mathrm{C} \mathrm{NMR}\left(100.6 \mathrm{MHz}, \mathrm{CDCl}_{3}\right): \delta_{\mathrm{C}}=$ $14.7(\mathrm{Me}), 19.2\left(\mathrm{CH}_{2}\right), 22.5\left(\mathrm{CH}_{2}\right), 22.9\left(\mathrm{CH}_{2}\right), 30.8(\mathrm{Me}), 31.6\left(\mathrm{CH}_{2}\right), 127.2(\mathrm{CH}), 128.1(2$ CH), $128.8(\mathrm{CH}), 129.7(\mathrm{CH}), 130.9(\mathrm{CH}), 131.4(2 \mathrm{CH}), 141.8(\mathrm{C}), 142.7(\mathrm{C}), 145.8(\mathrm{C}), 148.0$ (C), 160.7 (C), 164.9 (C). MS (EI, $70 \mathrm{eV}): m / z(\%)=384\left(\mathrm{M}^{+}, 5\right), 313$ (7), 293 (20), 229 (16), 155 (100), 91 (74), 77 (30), 76 (32), 71 (54). Anal. calcd. for $\mathrm{C}_{20} \mathrm{H}_{24} \mathrm{~N}_{4} \mathrm{O}_{2} \mathrm{~S}$ (384.16): C, 62.48; H, 6.29; N, 14.57\%. Found: C, 62.69; H, 6.33; N, 14.66\%.

$N$-(1H-benzo[d]imidazol-2-yl)-N'-(phenylsulfonyl)hexanimidamide 5e. Yield $0.21 \mathrm{~g}(57 \%)$; cream powder, m.p. $=119-122{ }^{\circ} \mathrm{C} . \mathrm{IR}(\mathrm{KBr}): 3348(\mathrm{NH}), 3066(\mathrm{NH}), 1451(\mathrm{C}=\mathrm{N})$, $1377\left(\mathrm{SO}_{2}\right), 1180 \mathrm{~cm}^{-1}\left(\mathrm{SO}_{2}\right) .{ }^{1} \mathrm{H} \mathrm{NMR}\left(400 \mathrm{MHz}, \mathrm{CDCl}_{3}\right): \delta_{\mathrm{H}}=0.89\left(3 \mathrm{H}, \mathrm{t},{ }^{3} J_{\mathrm{HH}}=6.9 \mathrm{~Hz}, \mathrm{Me}\right)$, 1.37-1.44 (2H, m, $\left.\mathrm{CH}_{2}\right), 1.46-1.54\left(2 \mathrm{H}, \mathrm{m}, \mathrm{CH}_{2}\right), 2.17-2.21\left(2 \mathrm{H}, \mathrm{m}, \mathrm{CH}_{2}\right), 3.39\left(2 \mathrm{H}, \mathrm{t},{ }^{3} J_{\mathrm{HH}}=6.9\right.$ $\left.\mathrm{Hz}, \mathrm{CH}_{2}\right), 7.61\left(2 \mathrm{H}, \mathrm{t},{ }^{3} J_{\mathrm{HH}}=7.9 \mathrm{~Hz}, \mathrm{Ar}\right), 7.70-7.78(2 \mathrm{H}, \mathrm{m}, \mathrm{Ph}), 7.98\left(1 \mathrm{H}, \mathrm{t},{ }^{3} J_{\mathrm{HH}}=7.5 \mathrm{~Hz}, \mathrm{Ar}\right)$, $8.06\left(2 \mathrm{H}, \mathrm{d},{ }^{3} J_{\mathrm{HH}}=7.9 \mathrm{~Hz}, \mathrm{Ar}\right), 8.13\left(1 \mathrm{H}, \mathrm{d},{ }^{3} J_{\mathrm{HH}}=7.5 \mathrm{~Hz}, \mathrm{Ar}\right), 8.40\left(1 \mathrm{H}, \mathrm{d},{ }^{3} J_{\mathrm{HH}}=7.5 \mathrm{~Hz}, \mathrm{Ar}\right)$, $9.51(1 \mathrm{H}, \mathrm{s}, \mathrm{NH}), 10.33(1 \mathrm{H}, \mathrm{s}, \mathrm{NH}) .{ }^{13} \mathrm{C}$ NMR $\left(100.6 \mathrm{MHz}, \mathrm{CDCl}_{3}\right): \delta_{\mathrm{C}}=14.7(\mathrm{Me}), 19.3$ $\left(\mathrm{CH}_{2}\right), 22.9\left(\mathrm{CH}_{2}\right), 30.8\left(\mathrm{CH}_{2}\right), 31.7\left(\mathrm{CH}_{2}\right), 127.2(2 \mathrm{CH}), 128.5(2 \mathrm{CH}), 129.3(\mathrm{CH}), 129.6$ $(\mathrm{CH}), 130.4(\mathrm{CH}), 131.7(\mathrm{CH}), 136.5(\mathrm{C}), 139.4(\mathrm{CH}), 144.0(\mathrm{C}), 145.4(\mathrm{C}), 162.0(\mathrm{C}), 166.8$ 
(C). MS (EI, $70 \mathrm{eV}): m / z(\%)=370\left(\mathrm{M}^{+}, 7\right), 299$ (17), 253 (20), 229 (34), 141 (100), 125 (23),

77 (41), 76 (33). Anal. calcd. for $\mathrm{C}_{19} \mathrm{H}_{22} \mathrm{~N}_{4} \mathrm{O}_{2} \mathrm{~S}$ (370.15): C, 61.60; H, 5.99; N, 15.12\%. Found: C, 61.21; H, 6.04; N, 15.21\%.

$N$-(1H-benzo[d]imidazol-2-yl)-N'-(methylsulfonyl)hexanimidamide $5 f . \quad$ Yield $0.16 \mathrm{~g}(53 \%)$; cream powder, m.p. $=100-103{ }^{\circ} \mathrm{C} . \mathrm{IR}(\mathrm{KBr}): 3387(\mathrm{NH}), 3043(\mathrm{NH}), 1449(\mathrm{C}=\mathrm{N})$, $1376\left(\mathrm{SO}_{2}\right), 1181 \mathrm{~cm}^{-1}\left(\mathrm{SO}_{2}\right) .{ }^{1} \mathrm{H} \mathrm{NMR}\left(400 \mathrm{MHz}, \mathrm{CDCl}_{3}\right): \delta_{\mathrm{H}}=0.90\left(3 \mathrm{H}, \mathrm{t},{ }^{3} J_{\mathrm{HH}}=6.9 \mathrm{~Hz}, \mathrm{Me}\right)$, 1.35-1.42 (2H, m, $\left.\mathrm{CH}_{2}\right), 1.44-1.50\left(2 \mathrm{H}, \mathrm{m}, \mathrm{CH}_{2}\right), 2.11-2.18\left(2 \mathrm{H}, \mathrm{m}, \mathrm{CH}_{2}\right), 3.10(3 \mathrm{H}, \mathrm{s}, \mathrm{Me}), 3.37$ $\left(2 \mathrm{H}, \mathrm{t},{ }^{3} J_{\mathrm{HH}}=6.9 \mathrm{~Hz}, \mathrm{CH}_{2}\right), 7.71\left(1 \mathrm{H}, \mathrm{t},{ }^{3} J_{\mathrm{HH}}=7.9 \mathrm{~Hz}, \mathrm{Ar}\right), 7.78\left(1 \mathrm{H}, \mathrm{t},{ }^{3} J_{\mathrm{HH}}=7.5 \mathrm{~Hz}, \mathrm{Ar}\right), 8.10$ $\left(1 \mathrm{H}, \mathrm{d},{ }^{3} J_{\mathrm{HH}}=7.9 \mathrm{~Hz}, \mathrm{Ar}\right), 8.40\left(1 \mathrm{H}, \mathrm{d},{ }^{3} J_{\mathrm{HH}}=7.5 \mathrm{~Hz}, \mathrm{Ar}\right), 9.21(1 \mathrm{H}, \mathrm{s}, \mathrm{NH}), 10.10(1 \mathrm{H}, \mathrm{s}, \mathrm{NH})$. ${ }^{13} \mathrm{C}$ NMR $\left(100.6 \mathrm{MHz}, \mathrm{CDCl}_{3}\right): \delta_{\mathrm{C}}=14.6(\mathrm{Me}), 19.2\left(\mathrm{CH}_{2}\right), 23.0\left(\mathrm{CH}_{2}\right), 30.7\left(\mathrm{CH}_{2}\right), 31.6\left(\mathrm{CH}_{2}\right)$, $33.0(\mathrm{Me}), 121.3(\mathrm{CH}), 124.4(\mathrm{CH}), 129.4(\mathrm{CH}), 130.4(\mathrm{CH}), 132.5(\mathrm{C}), 144.1(\mathrm{C}), 162.1(\mathrm{C})$, 165.5 (C). MS (EI, $70 \mathrm{eV}): m / z(\%)=308\left(\mathrm{M}^{+}, 7\right), 237$ (7), $229(20), 191(23), 176(20), 78$ (100), 77 (26). Anal. calcd. for $\mathrm{C}_{14} \mathrm{H}_{20} \mathrm{~N}_{4} \mathrm{O}_{2} \mathrm{~S}$ (308.13): C, 54.52; H, 6.54; N, 18.17\%. Found: C, $54.27 ; \mathrm{H}, 6.50 ; \mathrm{N}, 18.26 \%$.

$\mathbf{N}$-(1H-benzo[d]imidazol-2-yl)-N'-tosylpentanimidamide 5g. Yield 0.24 g (65\%); cream powder, m.p. $=102-105^{\circ} \mathrm{C} . \mathrm{IR}(\mathrm{KBr}): 3354(\mathrm{NH}), 3044(\mathrm{NH}), 1444(\mathrm{C}=\mathrm{N}), 1306\left(\mathrm{SO}_{2}\right)$, $1180 \mathrm{~cm}^{-1}\left(\mathrm{SO}_{2}\right) .{ }^{1} \mathrm{H}$ NMR $\left(400 \mathrm{MHz}, \mathrm{CDCl}_{3}\right): \delta_{\mathrm{H}}=1.02\left(3 \mathrm{H}, \mathrm{t},{ }^{3} J_{\mathrm{HH}}=6.9 \mathrm{~Hz}, \mathrm{Me}\right), 1.49-1.63$ $\left(2 \mathrm{H}, \mathrm{m}, \mathrm{CH}_{2}\right), 1.78-1.92\left(2 \mathrm{H}, \mathrm{m}, \mathrm{CH}_{2}\right), 2.50(3 \mathrm{H}, \mathrm{s}, \mathrm{Me}), 3.05\left(2 \mathrm{H}, \mathrm{t},{ }^{3} \mathrm{~J}_{\mathrm{HH}}=6.9 \mathrm{~Hz}, \mathrm{CH}_{2}\right), 7.41$ $\left(2 \mathrm{H}, \mathrm{d},{ }^{3} J_{\mathrm{HH}}=7.9 \mathrm{~Hz}, \mathrm{Ar}\right), 7.59\left(1 \mathrm{H}, \mathrm{t},{ }^{3} J_{\mathrm{HH}}=7.5 \mathrm{~Hz}, \mathrm{Ar}\right), 7.66\left(1 \mathrm{H}, \mathrm{t},{ }^{3} J_{\mathrm{HH}}=7.5 \mathrm{~Hz}, \mathrm{Ar}\right), 7.93$ $\left(2 \mathrm{H}, \mathrm{d},{ }^{3} J_{\mathrm{HH}}=7.9 \mathrm{~Hz}, \mathrm{Ar}\right), 8.04\left(1 \mathrm{H}, \mathrm{d},{ }^{3} J_{\mathrm{HH}}=7.5 \mathrm{~Hz}, \mathrm{Ar}\right), 8.30\left(1 \mathrm{H}, \mathrm{d},{ }^{3} J_{\mathrm{HH}}=7.5 \mathrm{~Hz}, \mathrm{Ar}\right), 8.62$ (1H, s, NH), $9.64(1 \mathrm{H}, \mathrm{s}, \mathrm{NH}) .{ }^{13} \mathrm{C} \mathrm{NMR}\left(100.6 \mathrm{MHz}, \mathrm{CDCl}_{3}\right): \delta_{\mathrm{C}}=14.5(\mathrm{Me}), 21.5\left(\mathrm{CH}_{2}\right), 22.4$ $\left(\mathrm{CH}_{2}\right), 23.0(\mathrm{Me}), 24.8\left(\mathrm{CH}_{2}\right), 123.8(\mathrm{CH}), 128.1(2 \mathrm{CH}), 128.4(\mathrm{CH}), 128.6(\mathrm{CH}), 128.7(\mathrm{CH})$, 130.1 (2 CH), 141.8 (C), 142.9 (C), 144.7 (C), 147.8 (C), 148.0 (C), 159.1 (C). MS (EI, 70 eV): 
$m / z(\%)=370\left(\mathrm{M}^{+}, 4\right), 253(9), 229(18), 155$ (100), 91 (36), 77 (38), 57 (32). Anal. calcd. for $\mathrm{C}_{19} \mathrm{H}_{22} \mathrm{~N}_{4} \mathrm{O}_{2} \mathrm{~S}$ (370.15): C, 61.60; H, 5.99; N, 15.12\%. Found: C, 62.09; H, 6.04; N, 15.26\%.

$N$-(1H-benzo[d]imidazol-2-yl)-N'-(phenylsulfonyl)pentanimidamide $5 \mathrm{~h}$. Yield $0.22 \mathrm{~g},(62 \%)$; cream powder, m.p. $=98-100^{\circ} \mathrm{C} . \mathrm{IR}(\mathrm{KBr}): 3333(\mathrm{NH}), 3098(\mathrm{NH}), 1404(\mathrm{C}=\mathrm{N})$, $1387\left(\mathrm{SO}_{2}\right), 1144 \mathrm{~cm}^{-1}\left(\mathrm{SO}_{2}\right) .{ }^{1} \mathrm{H} \mathrm{NMR}\left(400 \mathrm{MHz}, \mathrm{CDCl}_{3}\right): \delta_{\mathrm{H}}=0.98\left(3 \mathrm{H}, \mathrm{t},{ }^{3} J_{\mathrm{HH}}=6.9 \mathrm{~Hz}, \mathrm{Me}\right)$, 1.51-1.57 (2H, m, $\left.\mathrm{CH}_{2}\right), 2.14-2.23\left(2 \mathrm{H}, \mathrm{m}, \mathrm{CH}_{2}\right), 2.52\left(2 \mathrm{H}, \mathrm{t},{ }^{3} \mathrm{~J}_{\mathrm{HH}}=6.9 \mathrm{~Hz}, \mathrm{CH}_{2}\right), 7.64(2 \mathrm{H}, \mathrm{t}$, $\left.{ }^{3} J_{\mathrm{HH}}=7.9 \mathrm{~Hz}, \mathrm{Ar}\right), 7.70-7.75(2 \mathrm{H}, \mathrm{m}, \mathrm{Ph}), 7.96\left(1 \mathrm{H}, \mathrm{t},{ }^{3} J_{\mathrm{HH}}=7.5 \mathrm{~Hz}, \mathrm{Ar}\right), 8.01\left(2 \mathrm{H}, \mathrm{d},{ }^{3} J_{\mathrm{HH}}=7.9\right.$ $\mathrm{Hz}, \mathrm{Ar}), 8.08\left(1 \mathrm{H}, \mathrm{d},{ }^{3} J_{\mathrm{HH}}=7.5 \mathrm{~Hz}, \mathrm{Ar}\right), 8.37\left(1 \mathrm{H}, \mathrm{d},{ }^{3} \mathrm{~J}_{\mathrm{HH}}=7.5 \mathrm{~Hz}, \mathrm{Ar}\right), 9.31(1 \mathrm{H}, \mathrm{s}, \mathrm{NH}), 9.87$ $(1 \mathrm{H}, \mathrm{s}, \mathrm{NH}) .{ }^{13} \mathrm{C}$ NMR $\left(100.6 \mathrm{MHz}, \mathrm{CDCl}_{3}\right): \delta_{\mathrm{C}}=14.5(\mathrm{Me}), 21.5\left(\mathrm{CH}_{2}\right), 23.0\left(\mathrm{CH}_{2}\right), 26.2$ $\left(\mathrm{CH}_{2}\right), 121.7(2 \mathrm{CH}), 124.1(2 \mathrm{CH}), 127.1(\mathrm{CH}), 128.0(\mathrm{CH}), 130.4(\mathrm{CH}), 131.6(\mathrm{CH}), 138.7$ (CH), $131.6(\mathrm{C}), 132.9(\mathrm{C}), 136.5(\mathrm{C}), 145.6(\mathrm{C}), 160.1(\mathrm{C}) . \mathrm{MS}(\mathrm{EI}, 70 \mathrm{eV}): m / z(\%)=356\left(\mathrm{M}^{+}\right.$, 10), 299 (15), 224 (28), 132 (44), 141 (100), 125 (23), 77 (25), 76 (30). Anal. calcd. for $\mathrm{C}_{18} \mathrm{H}_{20} \mathrm{~N}_{4} \mathrm{O}_{2} \mathrm{~S}$ (356.13): C, 60.65; H, 5.66; N, 15.72\%. Found: C, 61.02; H, 5.70; N, 15.81\%. 
$N$-(1H-benzo[d]imidazol-2-yl)-2-phenyl-N'-tosylacetamidine (5a):
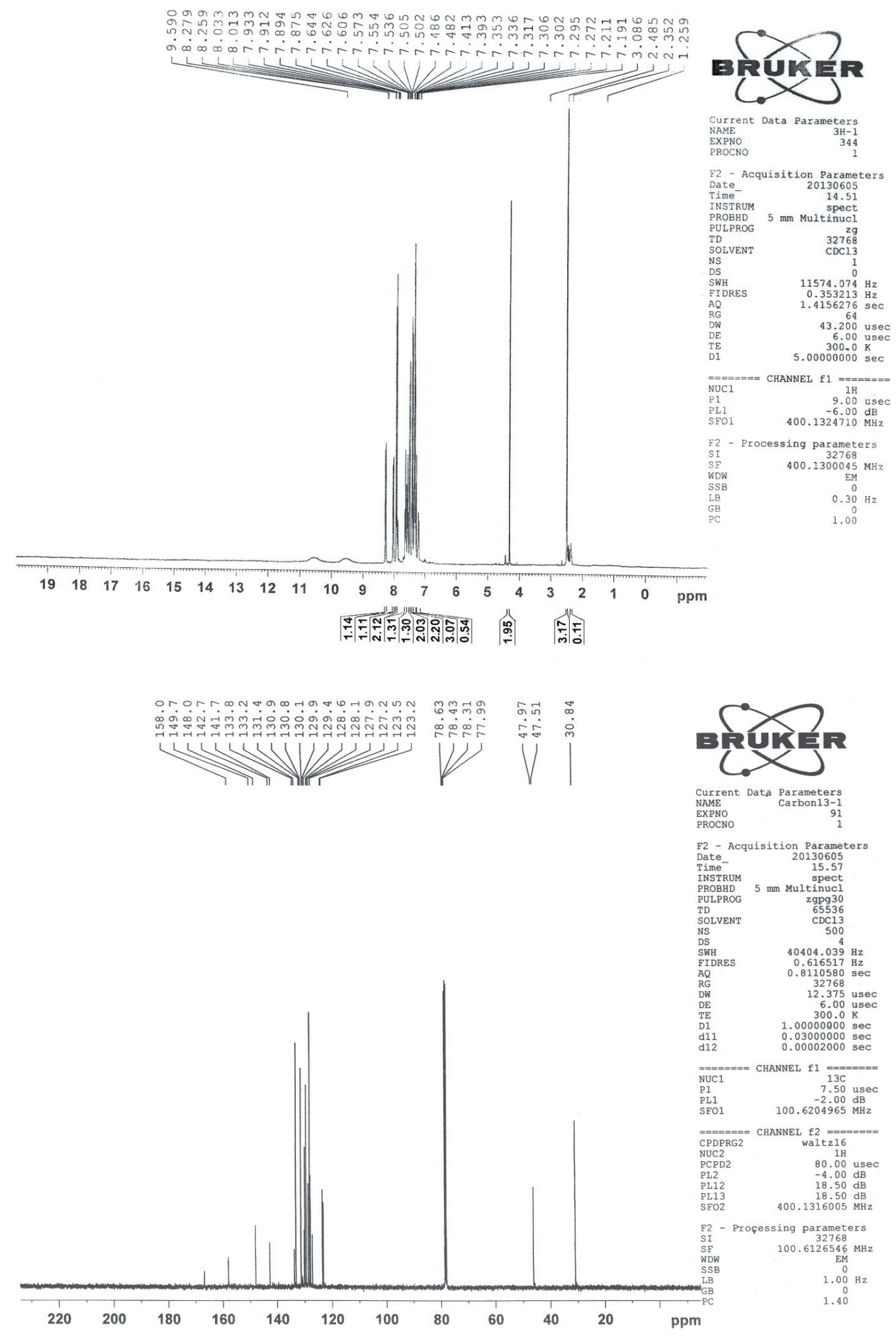

$N$-(1 H-benzo[d]imidazol-2-yl)-2-phenyl-N'-(phenylsulfonyl)acetimidamide (5b): 

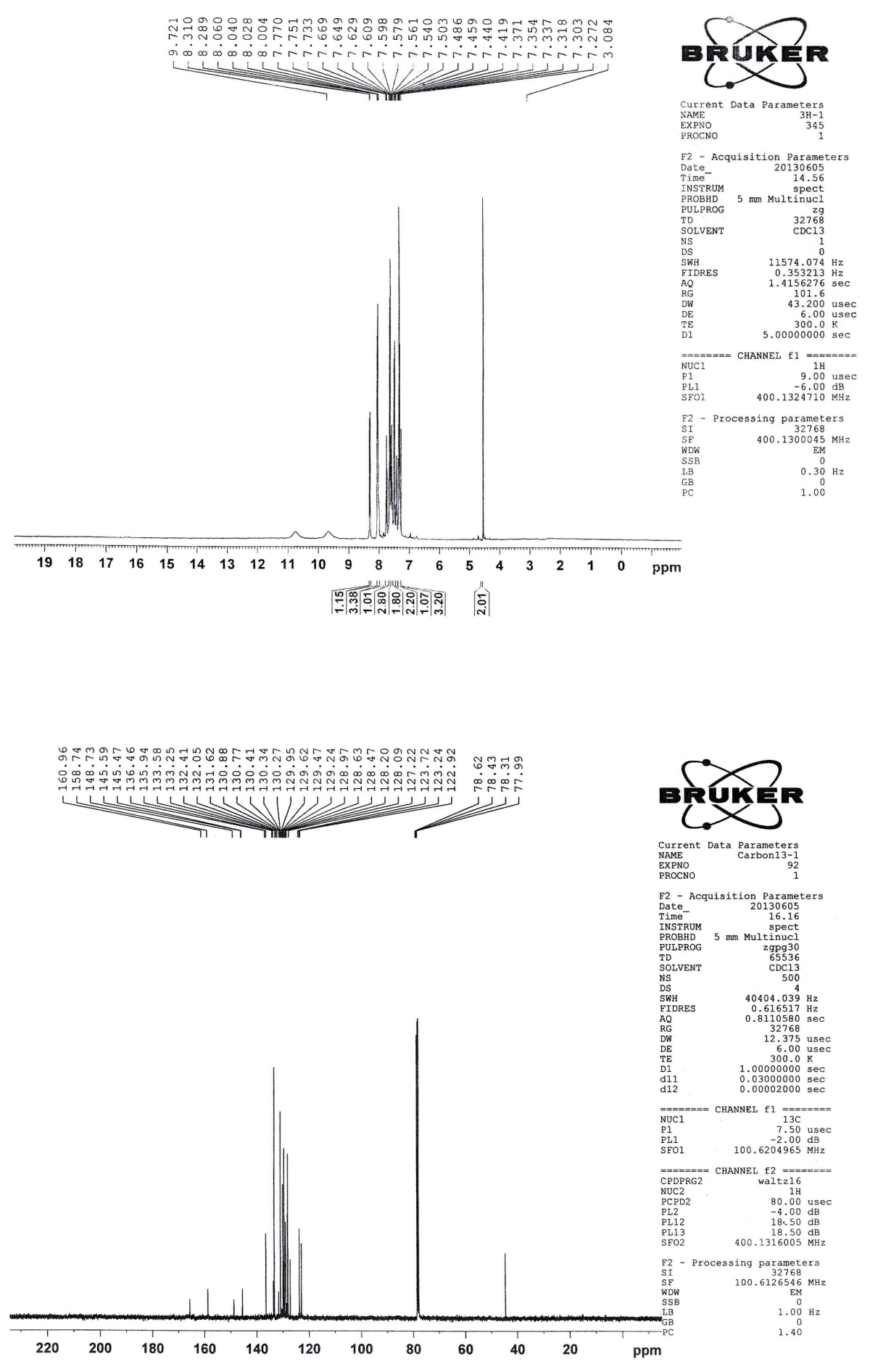

$N$-(1H-benzo[d]imidazol-2-yl)-N'-(methylsulfonyl)-2-phenylacetimidamide (5c): 

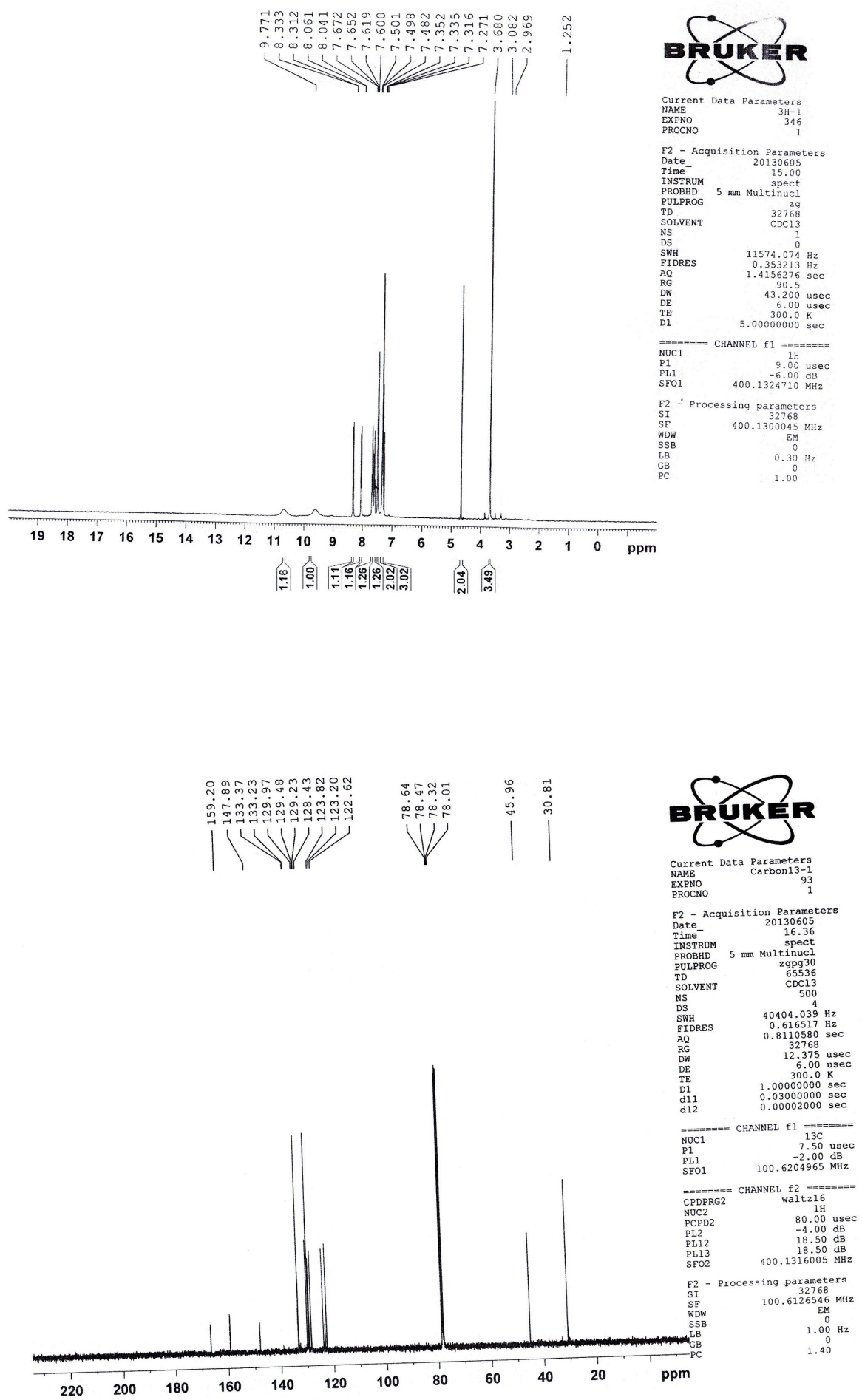

$N$-(1H-benzo[d]imidazol-2-yl)-N'-tosylhexanamidine (5d): 

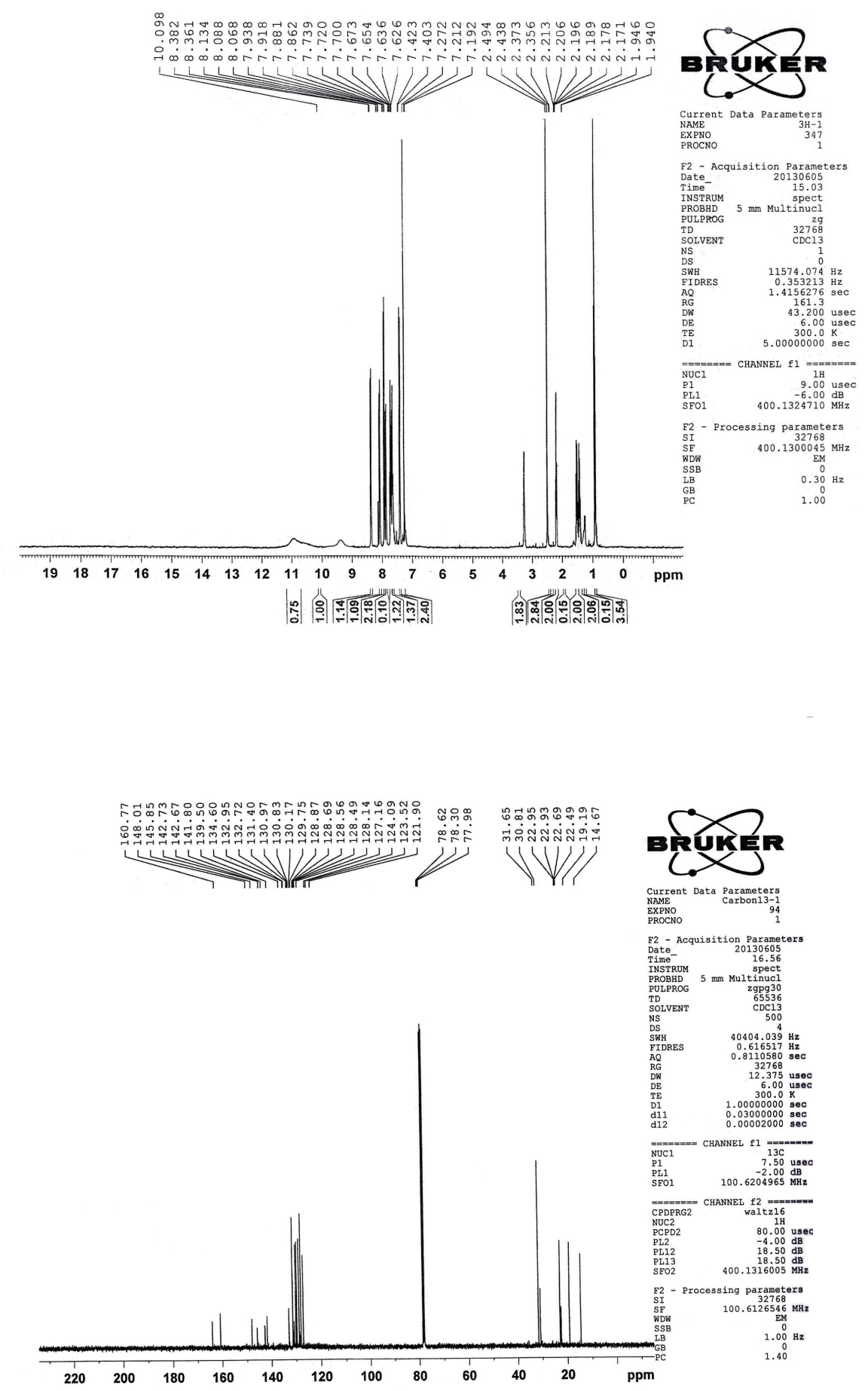

$N$-(1H-benzo[d]imidazol-2-yl)-N'-(phenylsulfonyl)hexanimidamide (5e): 

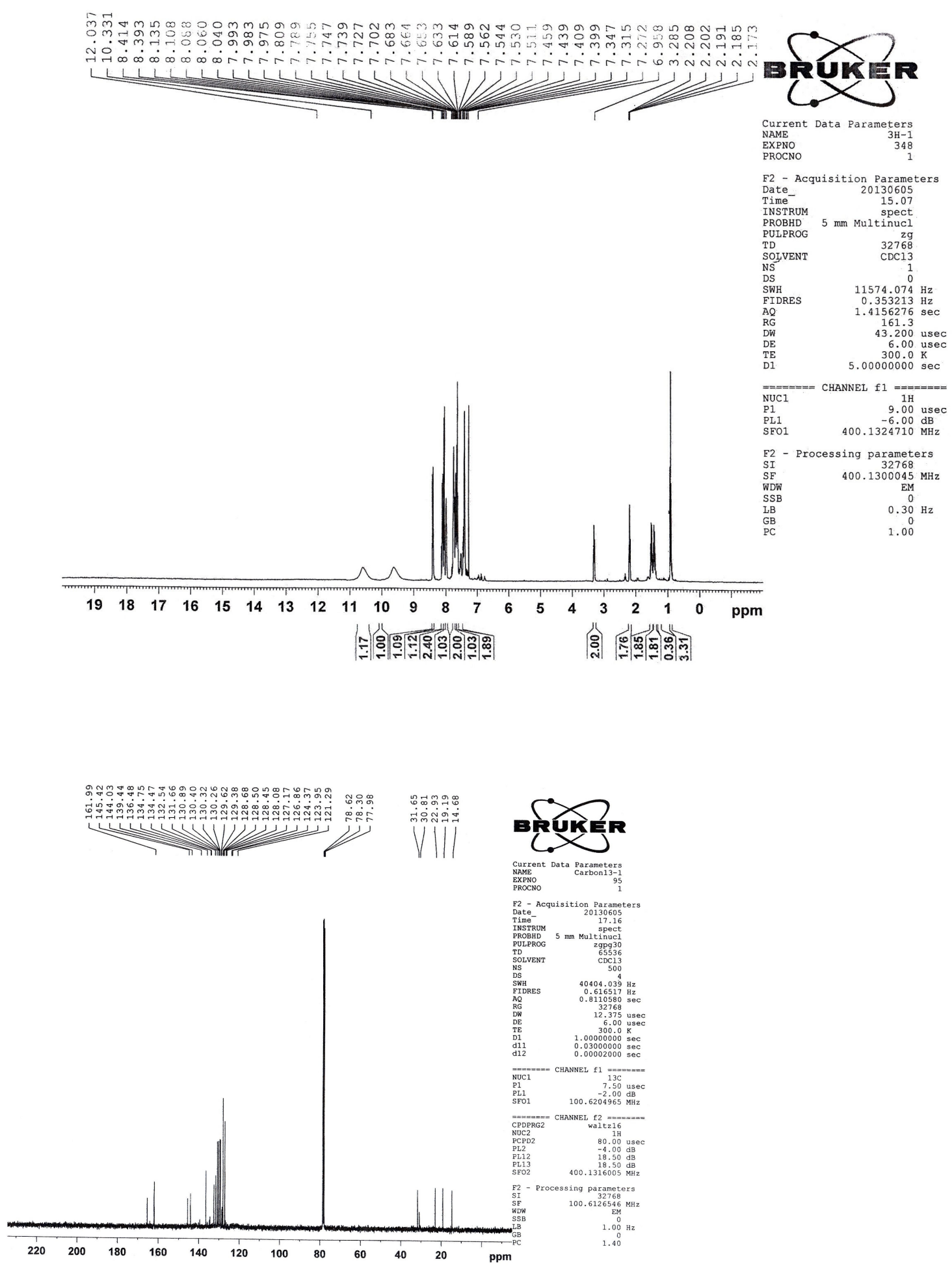

$N$-(1H-benzo[d]imidazol-2-yl)-N'-(methylsulfonyl)hexanimidamide (5f): 

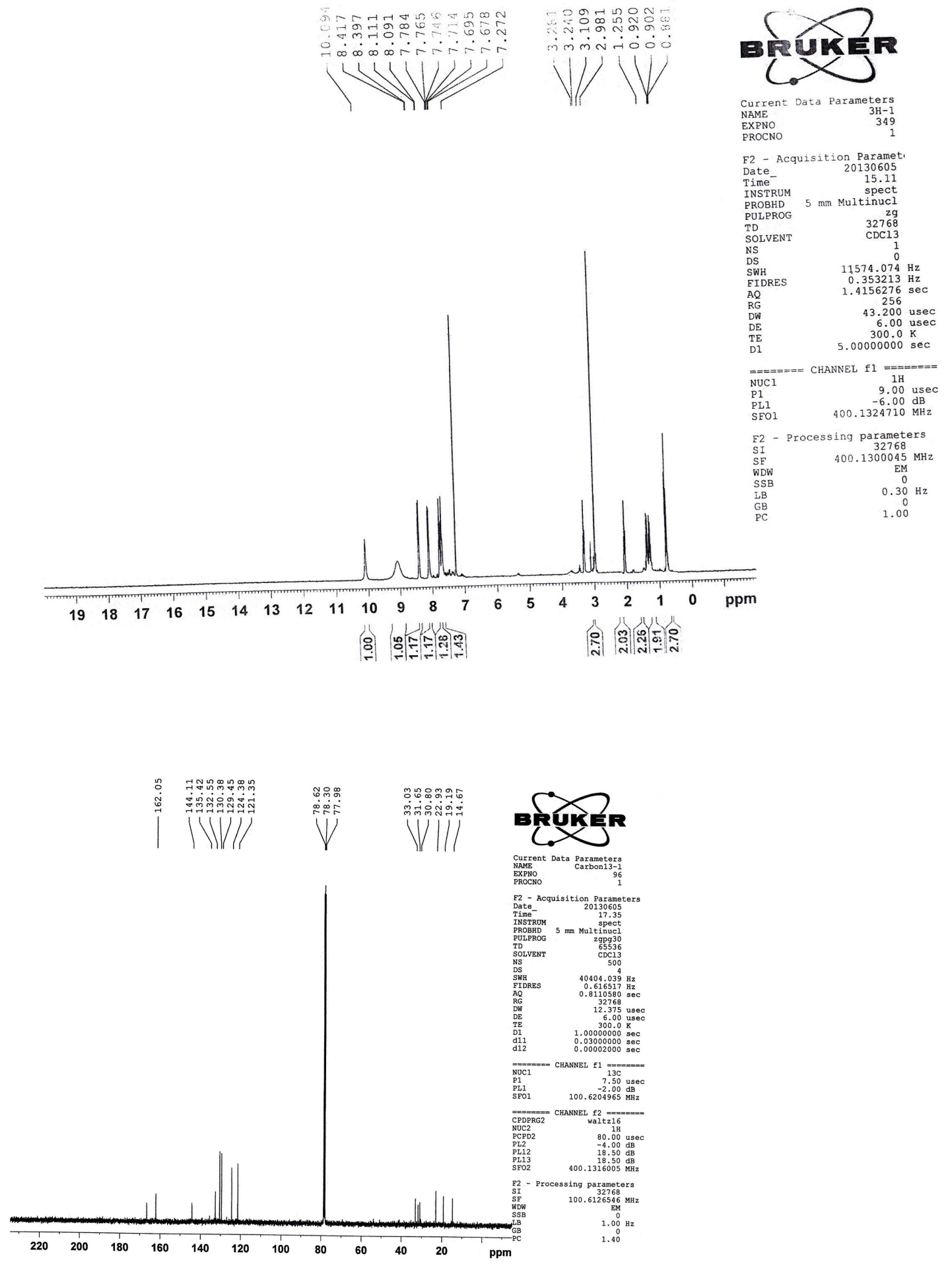

$N$-(1H-benzo[d]imidazol-2-yl)-N'-tosylpentanimidamide (5g): 

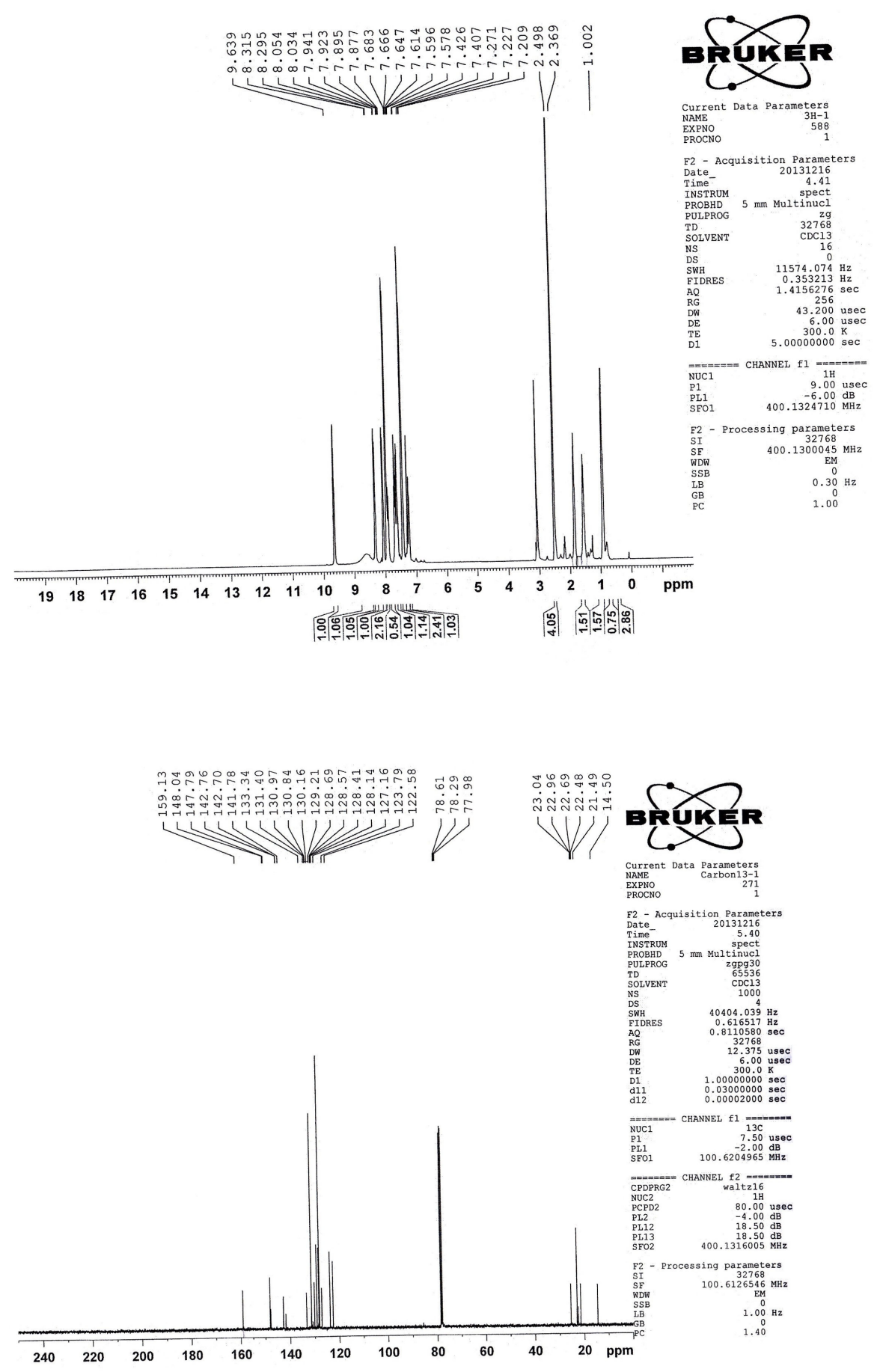

$N$-(1H-benzo[d]imidazol-2-yl)-N'-(phenylsulfonyl)pentanimidamide (5h): 

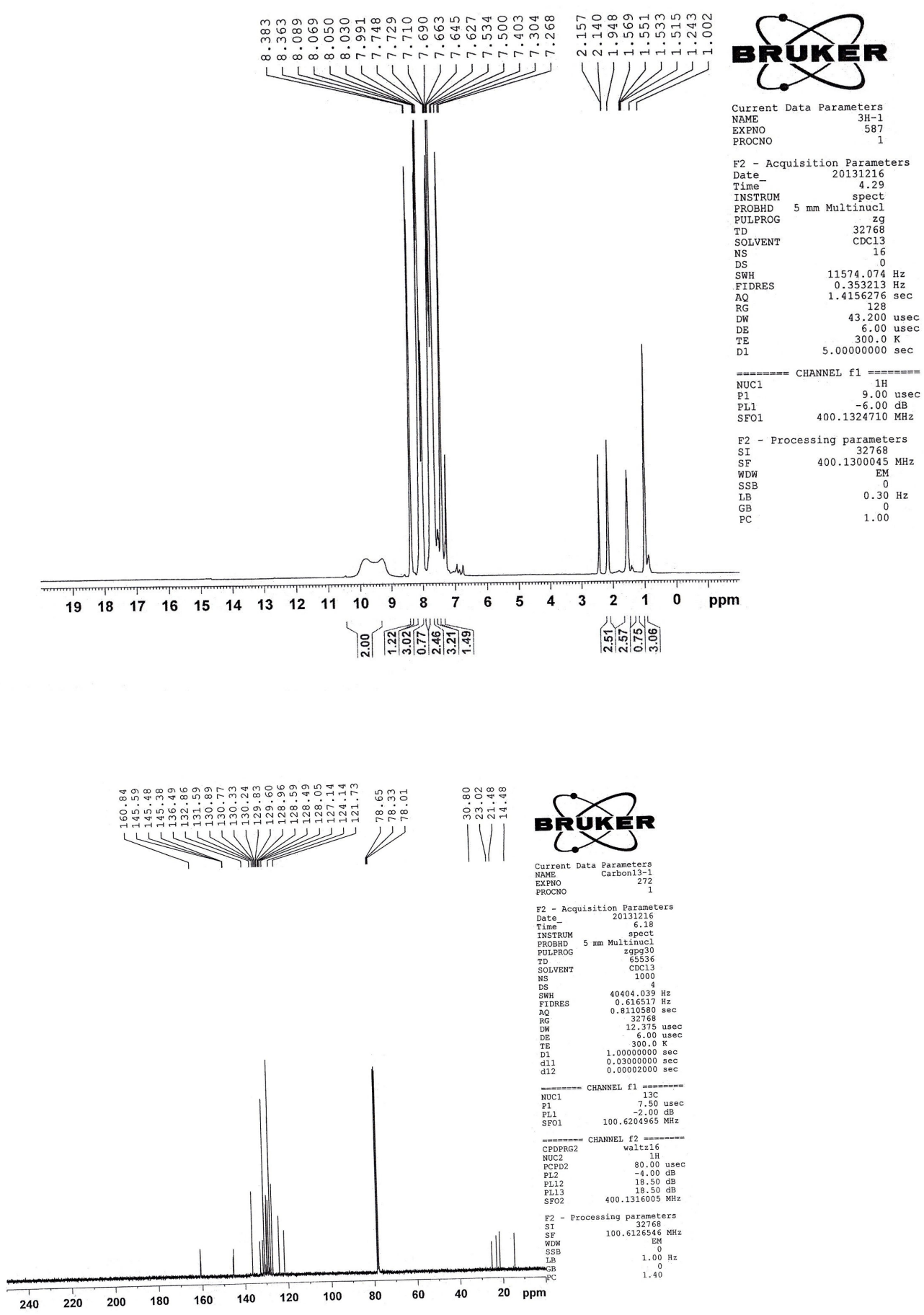